\title{
Effects of dietary cholesterol and fat on serum non-cholesterol sterols according to different apolipoprotein E subgroups among healthy men
}

\author{
Markku J. Nissinen ${ }^{1 *}$, Helena Gylling ${ }^{2}$ and Tatu A. Miettinen ${ }^{3}$ \\ ${ }^{1}$ Division of Gastroenterology, Department of Medicine, University of Helsinki, C422, POB 700, Helsinki FI-00029, Finland \\ ${ }^{2}$ Department of Clinical Nutrition, University of Kuopio and Kuopio University Hospital, Kuopio, Finland \\ ${ }^{3}$ Division of Internal Medicine, Department of Medicine, University of Helsinki, Helsinki, Finland
}

(Received 20 July 2007 - Revised 5 November 2007 - Accepted 14 November 2007 - First published online 18 February 2008)

The impact of apo E phenotypes on applicability of relative cholesterol synthesis (lathosterol:cholesterol) and absorption (ratios of cholestanol, campesterol and sitosterol to cholesterol) during diets of various cholesterol and fat content is unclear. We examined and compared with each other both relative and absolute synthesis and absorption among twenty-nine men, of whom eight, nine and twelve had apo $\mathrm{E}$ phenotypes $2(2 / 2,2 / 3,2 / 4), 3(3 / 3)$ and $4(3 / 4,4 / 4)$, respectively. Serum lipids, lipoproteins, sterols and cholesterol metabolism were examined on four subsequent diets: high-cholesterol high-fat (home diet; HD), low-cholesterol low-fat (LCLF), high-cholesterol low-fat (HCLF) and low-cholesterol high-fat (LCHF). LDL-cholesterol (LDL-C) level was about $40 \%$ lower $(P<0.05)$ in apo E2 than apo E3 and E4 groups irrespective of dietary fat and cholesterol. Serum proportions of phytosterols were determined apo E-dependently on LCLF and HCLF, and those of lathosterol, cholestanol and campesterol were increased in apo E2 and E3 groups $(P<0.05$ for each $v$. HD). Serum proportion of sitosterol reflected almost consistently apo E phenotype ( $r$ range +0.308 to $+0.383 ; P$ range $0.214-0.011)$. Relative cholesterol synthesis and absorption reflected respective absolute values during each diet in the apo $\mathrm{E} 4$ group $(r$ range +0.713 to $+0.893 ; P<0.05$ for each), but only during HD $(r+0.594$; $P=0.015)$ in the apo E2 + E3 group. The consumption of a high amount of fat did not interfere with cholesterol metabolism or serum levels of LDL-C differently in apo E phenotypes. Surrogate sterol markers of cholesterol metabolism reflected absolute ones (especially in the apo E4 group) and apo E phenotypes despite variable amounts of dietary cholesterol and fat

Apolipoprotein E: Cholesterol: Non-cholesterol sterol metabolism: Phytosterols

Apo E phenotypes E2, E3 and E4 are involved in the homeostasis of cholesterol metabolism, in its elimination as bile acids, in the removal of chylomicron remnants and in the hepatic clearance of dietary fat ${ }^{(1-3)}$. In addition, apo E phenotypes and cholesterol absorption efficiency are interrelated, so that the $\varepsilon 4$ isoform is associated with enhanced and $\varepsilon 2$ isoform with low cholesterol absorption efficiency ${ }^{(2)}$. Interrelationship of apo $\mathrm{E}$ phenotypes with cholesterol absorption may in fact be a reason for the respective high and low levels of serum cholesterol. However, the association between apo E phenotypes and serum cholesterol level seems to be dependent on the amount of dietary fat and cholesterol. We have shown in our previous studies that during ad libitum home diet (HD) with high fat and cholesterol intake (38\% energy $(\mathrm{E} \%) / \mathrm{d}$ and $574 \mathrm{mg} / \mathrm{d}$, respectively), a positive association between apo $\mathrm{E}$ phenotypes and cholesterol absorption efficiency was present, but not any longer when the intakes of fat and cholesterol were reduced ${ }^{(3,4)}$. Furthermore, during low fat and cholesterol intake $(24 \mathrm{E} \% / \mathrm{d}, 208 \mathrm{mg} / \mathrm{d}$, respectively), the absorption efficiency and synthesis of cholesterol were apo E dependent, vanishing, however, after four-fold increase in cholesterol intake ${ }^{(5)}$. Accordingly, it seems evident that the interplay between apo E phenotypes and cholesterol metabolism is dependent on the dietary amount of cholesterol and fat, and this may be the reason why in some studies this association could not be shown ${ }^{(6,7)}$. It seems also evident that apo $\mathrm{E}$ phenotype regulates the sensitivity of serum total cholesterol (TC) and LDL-cholesterol (LDL-C) levels in response to the amount of dietary cholesterol and fat $^{(3-5)}$. Accordingly, we were interested in the interplay of apo $\mathrm{E}$ and extensively increased intake in dietary saturated fat on serum TC and LDL-C and absolute cholesterol metabolism.

From among small amounts of non-cholesterol sterols in serum and especially in LDL particles the most important are dietary phytosterols, cholestanol, a metabolite of cholesterol, and cholesterol precursor sterols ${ }^{(8-10)}$. In steady-state conditions, the non-cholesterol sterols reflect cholesterol metabolism such that cholestanol and the phytosterols campesterol and sitosterol reflect positively the fractional cholesterol absorption $^{(8-10)}$, whereas the cholesterol precursor sterols lathosterol and desmosterol parallel the changes in cholesterol synthesis $^{(8)}$ and the activity of hepatic HMG-CoA reductase

Abbreviations: E\%, \% energy; HCLF, high-cholesterol low-fat diet; HD, home diet; LCHF, low-cholesterol high-fat diet; LCLF, low-cholesterol low-fat diet;

LDL-C, LDL-cholesterol; TC, total cholesterol.

* Corresponding author: Dr Markku Nissinen, fax +35894717 1851, email markku.nissinen@dlc.fi 
under normal conditions ${ }^{(11)}$. Since the positive association of apo E phenotypes with cholesterol metabolism was weakened when modulating the intakes of fat and cholesterol ${ }^{(3-5)}$, we assumed that apo E phenotypes interfere also the metabolism of these serum non-cholesterol sterols, and the regulation is dependent on the amount of dietary fat and cholesterol.

Accordingly, the aims of the present study were to evaluate the effects of different apo E phenotypes on non-cholesterol sterol metabolism when diets with low and high cholesterol and fat contents are consumed by healthy hypercholesterolaemic middle-aged men. Furthermore, we aimed to reveal the sensitivity of these surrogate sterol markers of cholesterol metabolism to the respective absolute ones in variable dietary conditions in different apo E phenotypes.

\section{Subjects and methods}

\section{Study population}

Since the baseline characteristics of the study population have been reported in earlier studies ${ }^{(3,5,12)}$, only a short description is given here. The study population consisted of twenty-nine healthy male subjects. Their age (54 (SEM 1) years) was equal in the apo E phenotypes. The study population included eight individuals with the $\varepsilon 2$ allele (two with apo E2/2, five with apo E2/3 and one with apo E2/4; the apo E2 group), nine with the apo E3/3 phenotype (the apo E3 group) and twelve with the $\varepsilon 4$ allele (ten with apo E3/4 and two with apo E4/4; the apo E4 group). All subjects gave informed consent, and the study protocol had been accepted by the
Ethics Committee of the Department of Internal Medicine, Helsinki University Central Hospital.

\section{Experimental design}

Results concerning LDL kinetics, cholesterol metabolism, and serum lipids and lipoproteins during low-fat low-cholesterol and low-fat high-cholesterol diets have been published earlier ${ }^{(3,5)}$. Our previous study revealed metabolism of cholesterol and non-cholesterol sterols in this study population without apo E reference during the four dietary interventions ${ }^{(12)}$. The subjects were studied first at baseline on their normal diet (baseline HD) and during the following three separate 6-week dietary periods at the out-patient ward at least 3 months apart (Table 1). On HD, the average daily cholesterol intake was $574 \mathrm{mg}$, and $38 \%$ of energy was as fat. During the low-cholesterol low-fat diet (LCLF), dietary fat and cholesterol intakes were $24 \mathrm{E} \%$ and $200 \mathrm{mg} / \mathrm{d}$. During the high-cholesterol low-fat diet (HCLF), cholesterol $(890 \mathrm{mg} / \mathrm{d})$ was added to the diet as three egg yolks per d, and the fat intake was $30 \mathrm{E} \%$. During the low-cholesterol high-fat diet (LCHF), $39 \%$ of energy was as fat and cholesterol intake was $200 \mathrm{mg} / \mathrm{d}$. Eighteen of the study subjects attended the fourth dietary period. Their relative distribution into different apo E groups was similar to that of the whole study group (i.e. six, five and seven subjects, respectively). On HD, three of the subjects refused to make stool collections and participate in cholesterol absorption measurement. The participants received written dietary instructions and oral advice from our dietitian on a weekly basis. During 1 week of each study period (the last week), the subjects carefully

Table 1. Baseline characteristics of study subjects according to apolipoprotein E subgroup

(Mean values with their standard errors)

\begin{tabular}{|c|c|c|c|c|c|c|c|c|c|}
\hline \multirow{2}{*}{$\begin{array}{l}\text { Diets... } \\
\text { Variables }\end{array}$} & \multicolumn{2}{|c|}{$\mathrm{HD}(n 29)$} & \multicolumn{2}{|c|}{ LCLF (n 29) } & \multicolumn{2}{|c|}{ HCLF (n 29) } & \multicolumn{2}{|c|}{$\operatorname{LCHF}(n 18)^{*}$} & \multirow[b]{2}{*}{$P$} \\
\hline & Mean & SEM & Mean & SEM & Mean & SEM & Mean & SEM & \\
\hline \multicolumn{10}{|c|}{ Dietary cholesterol (mg/kg per d) } \\
\hline Apo E2 ( $(n)$ & $7 \cdot 1$ & 0.8 & $2 \cdot 7 \dagger$ & 0.2 & $11.8 \ddagger$ & 0.5 & $4 \cdot 2 \S$ & 0.4 & $<0.001$ \\
\hline Apo E3 (n 9) & $6 \cdot 8$ & 0.5 & $2 \cdot 7 \dagger$ & 0.4 & $11 \cdot 1 \ddagger$ & $1 \cdot 3$ & $4 \cdot 3 \S$ & 0.7 & $<0.001$ \\
\hline Apo E4 ( $n$ 12) & $7 \cdot 7$ & 0.8 & $2.5 \dagger$ & 0.3 & $11.0 \ddagger$ & $1 \cdot 0$ & $3.8 \S$ & 0.5 & $<0.001$ \\
\hline$P$ & NS & & NS & & NS & & NS & & \\
\hline \multicolumn{10}{|c|}{ Dietary fat $(\mathrm{g} / \mathrm{kg}$ per $\mathrm{d})$} \\
\hline Apo E2 $(n 8)$ & 1.4 & 0.1 & $0.7 \dagger$ & 0.1 & $0.8 \dagger$ & 0.1 & $1 \cdot 1 \ddagger$ & 0.1 & $<0.001$ \\
\hline Apo E3 (n 9) & 1.5 & 0.1 & $0.7 \dagger$ & 0.1 & $1.0 \ddagger$ & 0.1 & $1.5 \ddagger$ & 0.2 & $<0.001$ \\
\hline Apo E4 (n 12) & 1.4 & 0.1 & $0.6 \dagger$ & 0.1 & $0.9 \dagger \ddagger$ & 0.1 & $1 \cdot 2 \ddagger$ & 0.1 & $<0.001$ \\
\hline$P$ & NS & & NS & & NS & & NS & & \\
\hline \multicolumn{10}{|l|}{ Dietary fat (E\%) } \\
\hline ApoE2 (n 8) & $36 \cdot 1$ & $2 \cdot 2$ & $24.5 \dagger$ & $1 \cdot 2$ & 33.0‡ & $2 \cdot 2$ & $38 \cdot 8 \ddagger$ & 3.5 & 0.005 \\
\hline ApoE3 (n 9) & $40 \cdot 5$ & 1.9 & $24.9 \dagger$ & $2 \cdot 0$ & $30 \cdot 1 \dagger$ & 1.6 & $43 \cdot 1 \ddagger \S$ & 0.6 & $<0.001$ \\
\hline ApoE4 (n 12) & 37.4 & $1 \cdot 2$ & $23.0 \dagger$ & $1 \cdot 8$ & $27 \cdot 5 \dagger$ & $2 \cdot 0$ & $36 \cdot 8 \mp \S$ & $1 \cdot 6$ & $<0.001$ \\
\hline$P$ & NS & & NS & & NS & & NS & & \\
\hline \multicolumn{10}{|c|}{ Dietary campesterol (mg/kg per d) } \\
\hline Apo E2 ( $n 8)$ & 0.7 & 0.1 & 0.7 & 0.1 & 0.6 & 0.1 & $0 \cdot 6^{\mathrm{a}}$ & 0.1 & NS \\
\hline Apo E3 (n 9) & 0.9 & 0.2 & 0.7 & 0.1 & 0.7 & 0.1 & $1 \cdot 1^{b}$ & 0.1 & NS \\
\hline Apo E4 ( $n$ 12) & 0.7 & 0.1 & 0.7 & 0.1 & 0.7 & 0.1 & $0 \cdot 7^{\mathrm{a}}$ & 0.1 & NS \\
\hline$P$ & NS & & NS & & NS & & 0.018 & & \\
\hline
\end{tabular}

HD, home diet; LCLF, low-cholesterol low-fat diet; HCLF, high-cholesterol low-fat diet; LCHF, low-cholesterol high-fat diet; $\mathrm{E} \%$, percentage energy.

a,b Mean values within a column with unlike superscript letters are significantly different $(P<0.05)$

* Number of subjects in the apo $E$ phenotypes 2, 3 and 4 were 6,5 and 7, respectively.

$\dagger$ Mean value is significantly different from that for $\operatorname{HD}(P<0.05)$.

$\ddagger$ Mean value is significantly different from that for $\operatorname{LCLF}(P<0.05)$

$\S$ Mean value is significantly different from that for $\operatorname{HCLF}(P<0.05)$ 
recorded their $3 \mathrm{~d}$ dietary intakes according to instructions given by the dietitian. Body weight was measured during each study period. Blood samples were drawn after an overnight fast once at the beginning (not shown) and three times (from which mean values were calculated) during the last week of each dietary period for lipid, lipoprotein and sterol analyses. Cholesterol absorption, sterol balance and faecal data were studied at the end of each period.

\section{Determinations}

All analyses of serum and faecal samples were carried out from fresh samples immediately after the study.

Serum lipids, lipoproteins and sterols, and faecal fat, bile acids and neutral sterols were measured as described earlier ${ }^{(13-17)}$. Also faecal steroid and cholesterol absorption measurements were performed as described earlier ${ }^{(3,5)}$. For these purposes all subjects consumed during the dietary recording week (the last week of each dietary period) a capsule containing $\left[{ }^{14} \mathrm{C}\right]$ cholesterol, $\left[{ }^{3} \mathrm{H}\right]$ sitosterol and chromic oxide $\left(\mathrm{Cr}_{2} \mathrm{O}_{3}\right)$ with each major meal (breakfast, lunch and dinner) for $7 \mathrm{~d}$. A $3 \mathrm{~d}$ stool collection was performed during the last $3 \mathrm{~d}$ of the week. Because the recoveries of $\left[{ }^{3} \mathrm{H}\right]$ sitosterol and $\mathrm{Cr}_{2} \mathrm{O}_{3}$ were identical, the faecal flow value was corrected with the recovery value of $\mathrm{Cr}_{2} \mathrm{O}_{3}^{(18)}$. Fractional absorption for cholesterol was measured as the change in the ratio of the two isotopes ${ }^{(19)}$. The utilisation of radioactive tracers was considered to be completely safe in the setting of the present study. The stable isotopes would have given equal results as discussed in our previous study ${ }^{(12)}$. Children and women of childbearing age were not included in our studies. The stable isotopes are preferable in further metabolic studies due to lack of radioactivity. Cholesterol synthesis was calculated by using the sterol balance technique as the difference between the sum of faecal bile acids plus neutral sterols of cholesterol origin (cholesterol + coprostanol + coprostanone) and dietary cholesterol. The latter was calculated from the dietary records (see above $)^{(20)}$. Synthesis of cholesterol $(\mathrm{mg} / \mathrm{d})$ and fractional cholesterol absorption (\%) were regarded as absolute markers of cholesterol synthesis and absorption, respectively.

Since plant sterols are not metabolised in the human body, and small amounts of absorbed dietary campesterol and sitosterol are secreted through bile into the intestine, indicates that their faecal amounts, including unchanged parent compounds and bacterial conversion products, represent their dietary intake. Faecal values of campesterol and sitosterol given in the present study include also their bacterial conversion products, and their respective sum represents dietary plant sterol intake.

The corresponding sterol markers for cholesterol absorption were ratios of cholestanol, campesterol and sitosterol to cholesterol in serum (called proportions in the text), whereas that of lathosterol served as a marker of cholesterol synthesis ${ }^{(8-10)}$. In the text proportions of absorption markers are called relative absorption and that of lathosterol relative synthesis. Ratios of relative synthesis to relative absorption (for example, lathosterol: sitosterol) were calculated to show synthesis of lathosterol in $\mu \mathrm{g}$ per $1 \mu \mathrm{g}$ sitosterol (or respective absorption sterol). Correspondingly, the absolute synthesis $(\mathrm{mg} / \mathrm{d})$ :absolute absorption $(\%)$ ratios were calculated to show amount of daily cholesterol synthesis per $1 \%$ of absolute cholesterol absorption.

The consumption of cholesterol, fat (Table 1), SFA, MUFA and PUFA was determined from the computerised data of the food records (not shown), and the PUFA:SFA ratio was calculated for each diet.

Apo E phenotyping was performed on serum samples by immunoelectrophoresis and electric focusing ${ }^{(1)}$.

\section{Statistical analysis}

The results are expressed as mean values with their standard errors. To eliminate the effect of varying cholesterol contents, serum non-cholesterol sterol values were related to serum cholesterol and are expressed in terms of $10^{2} \times \mathrm{mmol} / \mathrm{mol}$ cholesterol of the same GLC run (called proportions in the text). The data were analysed for significance and normality with Number Crunching Statistical Software ${ }^{\mathrm{TM}}$ (NCSS $^{\mathrm{TM}}$; Statistical Solutions Ltd, 2000, Kaysville, UT, USA). Non-parametric statistical tests were used, because variables were mainly not normally distributed and the number of study subjects in each apo E subgroup was small. Comparisons between the dietary periods and between the apo E subgroups within each dietary period were performed with Kruskall-Wallis non-parametric ANOVA. The $P$ values given by comparisons between the dietary periods are in the column on the right in Tables $1-3$. If the $P$ value was below 0.05 , comparisons between the diets were carried out with the Wilcoxon signed-rank test for difference in medians. Additionally, $P$ values for analysis between the apo E subgroups are given below the respective columns and comparisons between the subgroups were made accordingly if the $P$ value was below 0.05 . Correlations were analysed by the Spearman rank correlation test. The association of the apo $\mathrm{E}$ phenotypes with different variables or the changes in these variables was also tested by correlating the variables with the apo E phenotypes (termed apo $\mathrm{E}$ subscript in the text so that $\mathrm{E} 2 / 2=1, \mathrm{E} 2 / 3=2$, $\mathrm{E} 2 / 4=3, \mathrm{E} 3 / 3=4, \mathrm{E} 3 / 4=5$ and $\mathrm{E} 4 / 4=6$ ) according to the Spearman rank correlation test. To expand the range of variations the four dietary periods were combined and these data are given in the text as 'combined analysis' and in the tables as 'all'. Because the number of subjects, particularly in the apo E2 and E3 groups, was small and the most significant correlations were found in the apo E4 group, the E2 and E3 groups were combined in some correlation analysis to increase statistical power and for clarity of the data representation. A $P$ value $<0.05$ was considered significant.

\section{Results}

Diets

Body weight (81 (SEM 2) $\mathrm{kg}$ ) of the study subjects was equal between the apo E subgroups and remained stable during the dietary periods. Dietary cholesterol intake varied from 208 (SEM 13) $\mathrm{mg} / \mathrm{d}$ (LCLF) to 879 (SEM 39) $\mathrm{mg} / \mathrm{d}$ (HCLF), that of fat from 53 (SEM 3) g/d (LCLF) to 113 (SEM 4) g/d (HD) during the dietary periods, and mean $\mathrm{E} \%$ of fat was lowest on LCLF but highest on HD and LCHF (see Table 1 for statistical analysis), but these values were equal between the apo E subgroups within each diet (Table 1). Due to the high 
amount of saturated fat, the PUFA : SFA ratios were low on $\mathrm{HD}$ and LCHF, but equal between the apo $\mathrm{E}$ phenotypes within each diet (not shown).

Dietary intake of plant sterols was equal between the diets. Mean dietary campesterol intake varied from 52 (SEM 3) to 60 (SEM 6) $\mathrm{mg} / \mathrm{d}$, and the respective values for sitosterol were from 158 (SEM 12) to 174 (SEM 9) mg/d. Comparison between the apo E subgroups revealed higher intake of campesterol in the E3 group on LCHF (Table 1).

\section{Serum lipids and lipoproteins}

LCLF reduced mean serum TC level by $15 \%$ from HD in the E3 group $(P<0 \cdot 05)$. Comparison of TC between the subgroups showed higher TC values in the E3 and E4 groups than in the E2 group during HD and HCLF $(P<0.05$ for each). LDL-C was consistently lowest in the E2 group on each diet $(P<0.05$ for each) (Table 2$)$.

Mean serum levels of HDL-cholesterol and TAG remained unchanged during the dietary periods and between the apo $\mathrm{E}$ subgroups (data not shown).

\section{Non-cholesterol sterols}

Mean proportions of lathosterol in serum were higher during LCHF in the E2 group $(P<0.05$ for each diet $)$ and the E3 group $(P<0.05$ for LCLF and HCLF) than during any other diet (Table 2).

Mean proportions of serum cholestanol were elevated from HD during HCLF and LCHF in the E2 and E3 groups $(P<0.05$ for each) (Table 2$)$.

Serum proportion of campesterol was elevated on LCHF in the E2 and the E3 groups (Table 2). Comparisons between the apo E subgroups revealed higher mean proportions of both campesterol and sitosterol in the E3 and the E4 groups than in the E2 group on HCLF ( $P<0.05$ for each) (Table 2). A respective difference for sitosterol was also detected during LCLF ( $P<0.05$ for the E4 group) (Table 2). Amounts of dietary campesterol and sitosterol were positively associated with their respective proportions in serum in the combined apo E2 + E3 group during each diet $(r+0.502$ to +0.736 ; $P<0.05$ for each) (except campesterol during HD), but in the apo E4 group only during $\operatorname{LCLF}(r+0 \cdot 601$ to $+0 \cdot 664$; $P<0.05$ for both)

Table 2. Serum total and low-density lipoprotein-cholesterol, and non-cholesterol sterol proportions in study subjects during dietary periods (Mean values with their standard errors)

\begin{tabular}{|c|c|c|c|c|c|c|c|c|c|}
\hline \multirow{2}{*}{$\begin{array}{l}\text { Diets... } \\
\text { Variables }\end{array}$} & \multicolumn{2}{|c|}{$\mathrm{HD}(n 29)$} & \multicolumn{2}{|c|}{ LCLF (n 29) } & \multicolumn{2}{|c|}{ HCLF (n 29) } & \multicolumn{2}{|c|}{$\operatorname{LCHF}(n 18)^{*}$} & \multirow[b]{2}{*}{$P$} \\
\hline & Mean & SEM & Mean & SEM & Mean & SEM & Mean & SEM & \\
\hline \multicolumn{10}{|l|}{ Total cholesterol† } \\
\hline Apo E2 (n 8) & $5 \cdot 2^{a}$ & 0.4 & 4.4 & 0.5 & $4 \cdot 6^{a}$ & 0.3 & 4.9 & 0.4 & NS \\
\hline Apo E3 $(n 9)$ & $6 \cdot 6^{\mathrm{b}}$ & 0.2 & $5 \cdot 6 \ddagger$ & 0.3 & $6 \cdot 1^{\mathrm{b}}$ & 0.3 & 5.9 & 0.2 & 0.049 \\
\hline Apo E4 (n 12) & $6 \cdot 2^{b}$ & 0.4 & $5 \cdot 2$ & 0.3 & $5 \cdot 9^{b}$ & 0.3 & $5 \cdot 9$ & 0.5 & NS \\
\hline$P$ & 0.012 & & 0.050 & & 0.004 & & 0.138 & & \\
\hline \multicolumn{10}{|l|}{ LDL-cholesterol } \\
\hline Apo E2 (n 8) & $2 \cdot 3^{a}$ & 0.3 & $1.9^{\mathrm{a}}$ & 0.2 & $2 \cdot 0^{a}$ & 0.3 & $2 \cdot 0^{\mathrm{a}}$ & 0.3 & NS \\
\hline Apo E3 (n9) & $3 \cdot 8^{\mathrm{b}}$ & 0.2 & $3 \cdot 2^{\mathrm{b}}$ & 0.3 & $3 \cdot 5^{\mathrm{b}}$ & 0.2 & $3.5^{\mathrm{b}}$ & 0.2 & NS \\
\hline Apo E4 ( $n$ 12) & $3 \cdot 6^{\mathrm{b}}$ & 0.3 & $2 \cdot 9^{b}$ & 0.2 & $3 \cdot 4^{\mathrm{b}}$ & 0.2 & $3 \cdot 2^{b}$ & 0.3 & NS \\
\hline$P$ & 0.007 & & 0.001 & & $<0.001$ & & 0.016 & & \\
\hline \multicolumn{10}{|l|}{ Lathosterol§ } \\
\hline Apo E2 ( $n 8)$ & 162 & 14 & 150 & 16 & 143 & 11 & $241 \neq \| \emptyset$ & 15 & 0.006 \\
\hline Apo E3 ( $n 9)$ & 139 & 19 & 119 & 13 & 104 & 11 & $206 \ddagger \| q$ & 16 & 0.008 \\
\hline Apo E4 (n 12) & 138 & 18 & 133 & 17 & 136 & 22 & 253 & 56 & NS \\
\hline$P$ & NS & & NS & & NS & & NS & & \\
\hline \multicolumn{10}{|l|}{ Cholestanol§ } \\
\hline Apo E2 (n 8) & 72 & 5 & 99 & 6 & $104 \ddagger$ & 5 & $127 \ddagger$ & 5 & $<0.001$ \\
\hline Apo E3 ( $n 9)$ & 87 & 12 & 129 & 11 & $122 \ddagger$ & 6 & $141 \ddagger$ & 17 & 0.018 \\
\hline Apo E4 ( $n 12)$ & 93 & 10 & 125 & 13 & 122 & 10 & 131 & 12 & NS \\
\hline$P$ & NS & & NS & & NS & & NS & & \\
\hline \multicolumn{10}{|l|}{ Campesterol§ } \\
\hline Apo E2 ( $(8)$ & 136 & 14 & 148 & 15 & $120^{a}$ & 11 & $201 \neq \emptyset$ & 16 & 0.013 \\
\hline Apo E3 $(n 9)$ & 165 & 20 & 183 & 18 & $176^{\mathrm{b}}$ & 19 & $281 \ddagger$ & 27 & 0.042 \\
\hline Apo E4 (n 12) & 202 & 29 & 207 & 30 & $196^{b}$ & 29 & 262 & 42 & NS \\
\hline$P$ & NS & & NS & & 0.027 & & NS & & \\
\hline \multicolumn{10}{|l|}{ Sitosterol§ } \\
\hline Apo E2 (n 8) & 88 & 13 & $104^{a}$ & 4 & $88^{a}$ & 5 & 115 & 10 & NS \\
\hline Apo E3 (n9) & 117 & 15 & $137^{\mathrm{a}, \mathrm{b}}$ & 10 & $122^{b}$ & 10 & 156 & 11 & NS \\
\hline Apo E4 (n 12) & 129 & 11 & $143^{\mathrm{b}}$ & 13 & $136^{\mathrm{b}}$ & 14 & 143 & 15 & NS \\
\hline$P$ & NS & & 0.031 & & 0.014 & & NS & & \\
\hline
\end{tabular}

HD, home diet; LCLF, low-cholesterol low-fat diet; HCLF, high-cholesterol low-fat diet; LCHF, low-cholesterol high-fat diet.

${ }_{a, b}$ Mean values within a column with unlike superscript letters are significantly different $(P<0.05)$.

* Number of subjects in the apo E phenotypes 2, 3 and 4 were 6, 5 and 7, respectively.

†Enzymic cholesterol.

$\mp$ Mean value is significantly different from that for $\mathrm{HD}(P<0.05)$

$\S 100 \times \mathrm{mmol} / \mathrm{mol}$ cholesterol.

$\|$ Mean value is significantly different from that for LCLF $(P<0.05)$.

ๆ Mean value is significantly different from that for $\operatorname{HCLF}(P<0.05)$. 


\section{Cholesterol absorption and metabolism}

The data of fractional cholesterol absorption and absolute cholesterol synthesis in the apo E phenotypes during HD, LCLF and HCLF have been reported previously ${ }^{(3,5)}$ (also see Discussion). During the high-fat feeding, fractional cholesterol absorption (\%) was 34.8 (SEM 4.2), 39.9 (SEM 2.1) and 38.1 (SEM 4.1) (NS), cholesterol synthesis $(\mathrm{mg} / \mathrm{kg} / \mathrm{d}$ ) was 11.4 (SEM 1.9), 9.9 (SEM 1.6) and 8.1 (SEM 1.2) (NS), and faecal neutral sterols $(\mathrm{mg} / \mathrm{kg} / \mathrm{d})$ were 8.4 (SEM 1.0), 9.1 (SEM 0.8) and 6.8 (SEM 0.4) (NS) in the apo E2, E3 and E4 phenotypes, respectively. Subjects in the apo E3 and E4 phenotypes had about $26 \%(P<0.05)$ lower fractional cholesterol absorption during the high-fat feeding than during HD, while cholesterol synthesis remained equal in the apo $\mathrm{E}$ phenotypes between the diets. Faecal neutral sterols during the high-fat feeding were $43-54 \%$ lower than during HCLF $(P<0.05$ for each phenotype). Bile acid synthesis remained equal between the apo $\mathrm{E}$ phenotypes on each diet and between the diets (not shown).

Correlations between serum non-cholesterol sterols, absolute markers of cholesterol metabolism and apo E subscript

In the apo E4 group, relative markers of cholesterol absorption were almost consistently, during LCLF, HCLF and LCHF, negatively related to the absolute cholesterol synthesis ( $r$ range -0.597 to $-0.964 ; P$ range 0.04 to $<0.001$ ) and serum proportion of lathosterol ( $r$ range -0.524 to $-0.964 ; P$ range 0.08 to $<0.001$ ), but less consistently (i.e. on LCLF and HCLF) positively to fractional cholesterol absorption $(r$ range +0.462 to $+0.734 ; P$ range $0 \cdot 10-0 \cdot 01)$. Serum proportion of lathosterol was positively related to absolute cholesterol synthesis also in an apo E4-dependent manner ( $r$ range +0.538 to $+0.821 ; P$ range $0.07-0.02$ ).

Absolute cholesterol synthesis - but not the respective relative sterol marker, lathosterol (not shown) - was negatively associated with apo E subscript during HD and in combined analysis (Table 3)

Among the relative (cholestanol, campesterol and sitosterol) and absolute markers of cholesterol absorption, positive association was detected with apo E subscript in each of them in combined analysis and also during HD except campesterol (data not shown) (Table 3). Additionally, both plant sterols were positively related to apo $\mathrm{E}$ subscript with HCLF, and sitosterol also with LCLF (Table 3).

Ratios of relative ( $\mu \mathrm{g}$ lathosterol/ $\mu \mathrm{g}$ sitosterol) and absolute markers of cholesterol synthesis and absorption were negatively associated with apo E subscript during HD and in combined analysis (Table 3 ).

In the apo E2+E3 group, the lathosterol: sitosterol ratio was positively related to the absolute cholesterol synthesis: fractional absorption ratio in combined analysis, but, in dietspecific analysis, solely during the simultaneous high-cholesterol high-fat feeding (Fig. 1 (A)). In the apo E4 group, a positive relationship was found during each diet (Fig. 1 (B)).

\section{Discussion}

The main new results of the present study were that (1) the effects of the apo E phenotype on the serum levels of LDL-C were prevailing contrary to that of various amounts of cholesterol and fat in diets. (2) The most impressive interaction between diet and genetic regulation (apo $\mathrm{E}$ phenotypes) on serum cholesterol and cholesterol metabolism could be observed during simultaneous high cholesterol and high saturated fat intake (HD), when fractional cholesterol absorption was higher in the apo E3 (49.8 (SEM 2.3) \%) and apo E4 groups $(55.1$ (SEM 4.3$) \%)$ than in the apo E2 group $(40 \cdot 2$ (SEM 2.8$) \%)(P=0.020)$, but synthesis of cholesterol was about $56 \%$ lower in the apo E3 and E4 groups than in the E2 group $(P=0 \cdot 041)^{(3,5)}$. (3) Serum surrogate sterol markers of cholesterol metabolism reflected absolute absorption and synthesis of cholesterol despite varying amounts of dietary cholesterol and fat - particularly in the apo E4 group. (4) Both absolute and relative markers of cholesterol absorption were almost consistently positively associated with the apo E subscript in combined analysis of the diets and during the highcholesterol high-fat diet (HD). (5) During HCLF, serum proportions of campesterol and sitosterol were higher among subjects with the E3 and E4 than with the E2 phenotype, probably mirroring higher fractional sterol absorption in these subgroups. (6) LCHF increased serum proportions of cholestanol, campesterol and sitosterol in the apo E2 and E3 groups. (7) Metabolism of lathosterol was apo E dependent.

It was the apo E4 group in which the relative sterol markers of cholesterol metabolism were almost consistently related to the respective absolute values during the different diets. Interestingly, during LCHF, when surprisingly high serum proportions of these non-cholesterol sterols were detected, lathosterol was tightly inversely interrelated to the three relative serum sterol markers of cholesterol absorption (cholestanol, campesterol and sitosterol). This finding indicates that despite the increase in serum proportions of these non-cholesterol sterols they reliably serve as surrogate markers of cholesterol metabolism. Our previous study showed that cholesterol homeostasis is maintained during consumption of various amounts of dietary cholesterol and fat, and that surrogate markers reliably follow-up the changes in cholesterol metabolism ${ }^{(12)}$. The results of the present study suggest that these serum non-cholesterol sterol markers were even more sensitive to reflect homeostasis of cholesterol metabolism during the diets - especially in the apo E4 group - than the absolute values, as the absolute ones showed weak interrelation with each other in the present apo E phenotypespecific analysis. The reason for the weak interrelationship of the absolute values with each other in the present study may be the small number of subjects in apo E phenotypes, because the interrelationship was consistent during the diets in the whole study population of twenty-nine subjects ${ }^{(12)}$. Results of our previous ${ }^{(12)}$ and the present study are in agreement with earlier studies concerning the applicability of cholestanol as a relative marker of cholesterol absorption ${ }^{(8-10,21)}$.

Among our study subjects, the absolute cholesterol synthesis and the absolute cholesterol synthesis:fractional cholesterol absorption ratio were inversely related to the apo $\mathrm{E}$ phenotype during $\mathrm{HD}$ and in combined analysis. Apo $\mathrm{E}$ phenotype-dependency was also found when the ratio of the corresponding surrogate markers - lathosterol:sitosterol was compared with the apo E subscript. Additionally, on HD and in combined analysis, fractional cholesterol absorption and serum proportion of cholestanol positively reflected the apo E subscript. Overall, these findings suggest that 
Table 3. Correlation matrices for apolipoprotein E subscript and relative and absolute markers of cholesterol absorption and synthesis during the home diet (HD) and after switching to a low-cholesterol low-fat diet (LCLF), a high-cholesterol low-fat diet (HCLF) and a low-cholesterol high-fat diet (LCHF) and in combined analysis

\begin{tabular}{|c|c|c|c|c|c|c|}
\hline $\begin{array}{l}\text { Variables... } \\
\text { Apo E subscript }\end{array}$ & $\begin{array}{l}\text { Cholesterol } \\
\text { synthesis } \\
\text { (mg/d) }\end{array}$ & $\begin{array}{l}\text { Cholestanol } \\
\text { proportion } \\
\text { (mmol/mol } \\
\text { cholesterol) }\end{array}$ & $\begin{array}{l}\text { Sitosterol } \\
\text { proportion } \\
\text { (mmol/mol } \\
\text { cholesterol) }\end{array}$ & $\begin{array}{l}\text { Cholesterol } \\
\text { absorption } \\
(\%)\end{array}$ & $\begin{array}{l}\text { Lathosterol:sitosterol } \\
(\mu \mathrm{g} \text { lathosterol } / \mu \mathrm{g} \\
\text { sitosterol }\end{array}$ & $\begin{array}{l}\text { Synthesis:absorption } \\
(\mathrm{mg} / \mathrm{d} / \%)\end{array}$ \\
\hline $\mathrm{HD}(n 27)$ & $-0.413^{\star}$ & $+0.400^{*}$ & $+0.383^{\star}$ & $+0.397^{\star}$ & $-0.469^{*}$ & $-0.514^{\star \star}$ \\
\hline $\operatorname{LCLF}(n 29)$ & -0.283 & +0.342 & $+0.428^{\star}$ & +0.183 & -0.365 & -0.318 \\
\hline $\operatorname{HCLF}(n 29)$ & -0.197 & +0.356 & $+0.463^{\star}$ & +0.301 & -0.314 & -0.195 \\
\hline LCHF ( $n$ 18) & -0.324 & +0.141 & +0.308 & +0.214 & -0.369 & -0.405 \\
\hline All (n 103) & $-0.285^{\star \star}$ & $+0.253^{*}$ & $+0.381^{\star \star \star}$ & $+0.239^{\star}$ & $-0 \cdot 349^{\star \star \star}$ & $-0.302^{\star \star}$ \\
\hline
\end{tabular}

${ }^{\star} P<0.05,{ }^{\star *} P<0.01,{ }^{* \star *} P<0.001$.

absolute and relative markers of cholesterol synthesis and absorption reflect diet-induced changes in cholesterol metabolism equally with respect to the apo E subscript.

Earlier data indicate that subjects with the apo E4 phenotype have higher fractional cholesterol absorption than those with apo E2, whereas the apo E3 phenotype falls in between $^{(2-5)}$. According to the absolute values, a trend similar to that was also found during LCHF in the present study. It has also been shown that reduction in the amount of dietary fat and cholesterol leads to disappearance of the relationship between fractional cholesterol absorption and the apo E phenotype ${ }^{(3,5)}$. Contrary to these results, a previous study performed with subjects homozygous for the apo E2 and E4 consuming $62-409 \mathrm{mg}$ cholesterol daily indicated that the efficiency of intestinal absorption and synthesis of cholesterol in humans is not related to the apo E phenotype ${ }^{(6)}$. In this respect, equal results were obtained by Sehayek et al. ${ }^{(7)}$.
Results of the present study support the view that apo E phenotype is involved in determining fractional cholesterol absorption. This was evidenced by the higher proportions of campesterol and particularly of sitosterol among the E3 and E4 groups than the E2 group especially during HCLF, but also to a lesser extent during LCLF. Furthermore, the present study showed a positive relationship of serum sitosterol proportion to apo E subscript on each diet except LCHF. However, the results of the present and the previous studies ${ }^{(6)}$ are not fully comparable as our study subjects included only two subjects homozygous for apo E2 and E4. A weakness of the present study was that only eighteen of the study subjects volunteered to attend the high-fat feeding diet, which led to a low number of study subjects in each apo E subtype, thus reducing the statistical power. That fractional cholesterol absorption decreased during each diet from HD in the E3 and E4 groups (also during LCHF as a new finding) is in
(A)

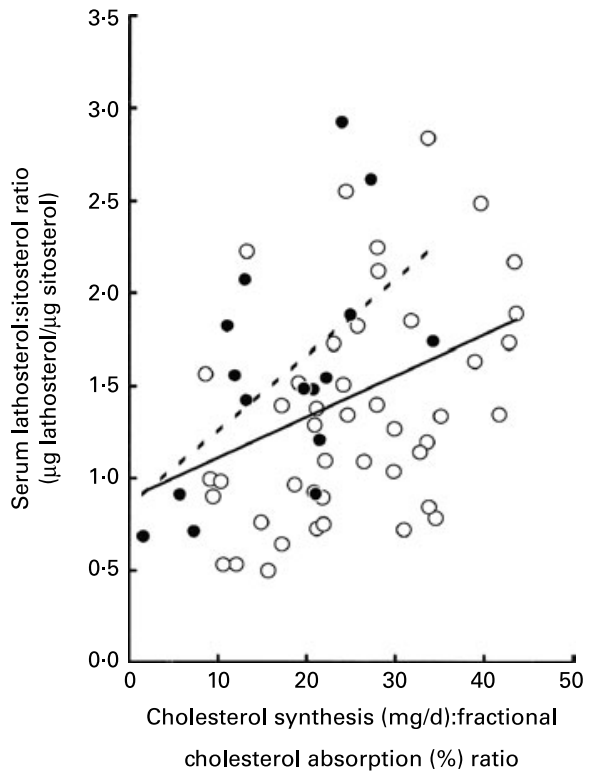

(B)

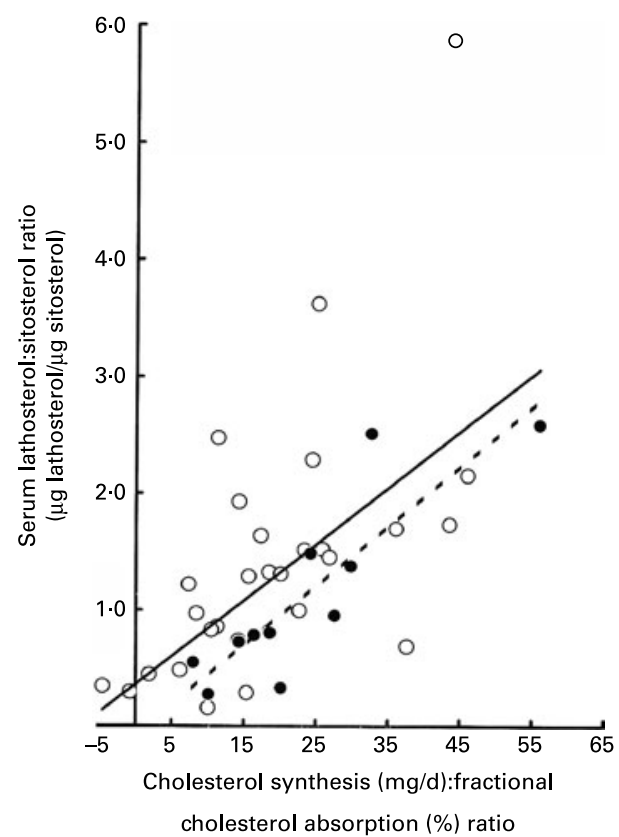

Fig. 1. Spearman rank correlations between serum lathosterol: sitosterol ratios ( $\mu$ g lathosterol/ $\mu \mathrm{g}$ sitosterol) and absolute cholesterol synthesis (mg/d):fractional cholesterol absorption (\%) ratio in the apo E2 + E3 group (A) and the apo E4 group (B) during combined analysis, in which all diets are included (-). Home diet $(\mathrm{HD})$ in (A) and low-cholesterol low-fat diet (LCLF) in (B) are illustrated separately $(--)$. (A) For diets combined for the apo E2 $+\mathrm{E} 3 \mathrm{group}(n 61$; $O$ ), $r$ 0.388, $P=0.002, y=0.02 x+0.89$. For the HD for the apo E2 + E3 group $(n 16 ; \bullet), r 0.594, P=0.015, y=0.04 x+0.85$. (B) For diets combined for the apo E4 group $(n 40 ; 0), r 0.659, P<0.001, y=0.05 x+0.36$. For LCLF for the apo E4 group $(n 12 ; \bullet), r 0.853, P<0.001, y=0.05 x-0.09$. 
accordance with earlier data suggesting that these phenotypes are more sensitive to changes in the amount of dietary cholesterol and fat than the E2.

One of the clinically relevant findings of the present study was that despite the amount of cholesterol and fat in the diet, serum proportions of plant sterols - particularly that of sitosterol were determined in an apo E phenotype-dependent manner. This was evidenced, first, by the high serum proportions of the two plant sterols in the apo E3 and E4 groups particularly during HCLF, and, second, by their positive relationship to apo E subscript. This may indicate that especially subjects with the $\varepsilon 4$ allele are at increased risk of the putative adverse effects of plant sterols, for example, premature atherosclerosis, as suggested in recent studies ${ }^{(22,23)}$. LCHF diet apparently could not inhibit plant sterol absorption. Interestingly, however, their serum proportions were almost consistently sensitive to their dietary amount in the apo E2 and E3 groups contrary to that of the apo E4 group. In our previous study, serum proportions of campesterol and sitosterol were increased during LCHF putatively due to their better absorption during dietary consumption of high amounts of saturated fat of plant origin ${ }^{(12)}$.

In conclusion, the present study indicated that a high amount of dietary fat does not interfere with cholesterol metabolism, serum levels of LDL-C or serum proportions of sitosterol and campesterol differently in the apo E phenotypes. Furthermore, despite the influence of the apo E phenotype on metabolism of non-cholesterol sterols, they serve as surrogate markers to detect changes in cholesterol metabolism during diets of different cholesterol and fat contents.

\section{Acknowledgements}

The study was supported by grants from the Paavo Nurmi Foundation and by a Helsinki University Hospital EVO grant. There are no conflicts of interest. Part of the results have been presented previously as an abstract by Gylling \& Miettinen $^{(24)}$.

\section{References}

1. Ehnholm C, Lukka M, Kuusi T, Nikkilä E \& Utermann G (1986) Apolipoprotein E polymorphism in the Finnish population: gene frequencies and relation to lipoprotein concentrations. J Lipid Res 27, 227-235.

2. Kesäniemi YA, Ehnholm C \& Miettinen TA (1987) Intestinal cholesterol absorption efficiency in man is related to apoprotein E phenotype. J Clin Invest 80, 578-581.

3. Miettinen TA, Gylling H, Vanhanen H \& Ollus A (1992) Cholesterol absorption, elimination, and synthesis related to LDL kinetics during varying fat intake in men with different apoprotein E phenotypes. Arterioscler Thromb 12, 1044-1052.

4. Miettinen TA, Gylling H \& Vanhanen H (1988) Serum cholesterol response to dietary cholesterol and apoprotein $\mathrm{E}$ phenotype. Lancet 8622, 1261.

5. Gylling H \& Miettinen TA (1992) Cholesterol absorption and synthesis related to low density lipoprotein metabolism during varying cholesterol intake in men with different apoE phenotypes. J Lipid Res 33, 1361-1371

6. von Bergmann K, Lütjohann D, Lindenthal B \& Steinmetz A (2003) Efficiency of intestinal cholesterol absorption in humans is not related to apoE phenotype. J Lipid Res 44, 193-197.
7. Sehayek E, Nath C, Heinemann T, McGee M, Seidman CE, Samuel P \& Breslow JL (1998) U-shape relationship between change in dietary cholesterol absorption in normal women and plasma lipoprotein responsiveness and evidence for extreme interindividual variation in dietary cholesterol absorption in humans. J Lipid Res 39, 2415-2422.

8. Miettinen TA, Tilvis RS \& Kesäniemi YA (1990) Serum plant sterols and cholesterol precursors reflect cholesterol absorption and synthesis in volunteers of a randomly selected male population. Am J Epidemiol 131, 20-31.

9. Miettinen TA, Tilvis RS \& Kesäniemi YA (1989) Serum cholestanol and plant sterol levels in relation to cholesterol metabolism in middle-aged men. Metabolism 38, 136-140.

10. Tilvis RS \& Miettinen TA (1986) Serum plant sterols and their relation to cholesterol absorption. Am J Clin Nutr 43, 92-97.

11. Björkhem I, Miettinen TA, Reihner E, Ewerth S, Angelin B \& Einarsson K (1987) Correlation between serum levels of some cholesterol precursors and activity of HMG-CoA reductase in human liver. J Lipid Res 28, 1137-1143.

12. Nissinen MJ, Gylling H \& Miettinen TA (2007) Responses of surrogate markers of cholesterol absorption and synthesis to changes in cholesterol metabolism during various amounts of fat and cholesterol feeding among healthy men. $\mathrm{Br} J$ Nutr (Epublication ahead of print version 15 August 2007).

13. Anonymous (1974) Manual of Laboratory Operations, Lipid Research Clinic Program: Lipid and Lipoprotein Analysis, DHEW Publication no. NIH/76-628.Washington, DC: Government Printing Office.

14. Miettinen TA (1988) Cholesterol metabolism during ketoconazole treatment in man. J Lipid Res 29, 43-51.

15. Miettinen TA \& Koivisto P (1983) Non-cholesterol sterols and bile acid production in hypercholesterolaemic patients with ileal by-pass. In Bile Acids and Cholesterol in Health and Disease, pp. 183-187 [G Paumgartner, A Stiehl and W Gerok, editors]. Lancaster, PA: MTP Press.

16. van de Kamer JH, Bokkel Huinink HT \& Weyers HA (1949) Rapid method for the determination of fat in feces. $J$ Biol Chem 177, 347-355.

17. Miettinen TA (1982) Gas-liquid chromatographic determination of fecal neutral sterols using capillary column. Clin Chim Acta 124, 245-248.

18. Bolin DW, King RP \& Klosterman EW (1952) A simplified method for the determination of chromic oxide $\left(\mathrm{Cr}_{2} \mathrm{O}_{3}\right)$ when used as an index substance. Science 116, 634-635.

19. Crouse JR \& Grundy SM (1978) Evaluation of a continuous isotope feeding method for measurement of cholesterol absorption in man. J Lipid Res 19, 221-232.

20. Ahlström A, Räsänen L \& Kuvaja K (1972) A method of data processing for food consumption surveys. Ann Acad Sci Fenn (Biol) 194, 1-8.

21. Gylling H, Vuoristo M, Färkkilä M \& Miettinen TA (1996) The metabolism of cholestanol in primary biliary cirrhosis. $J$ Hepatol 24, 444-451.

22. Assmann G, Cullen P, Erbey J, Ramey DR, Kannenberg F \& Schulte H (2005) Plasma sitosterol elevations are associated with an increased incidence of coronary events in men: results of a nested case-control analysis of the Prospective Cardiovascular Münster (PROCAM) study. Nutr Metab Cardiovasc Dis 16, 13-21.

23. Miettinen TA, Railo M, Lepäntalo M \& Gylling H (2005) Plant sterols in serum and in atherosclerotic plaques of patients undergoing carotid endarterectomy. $\mathrm{J} \mathrm{Am} \mathrm{Coll} \mathrm{Cardiol}$ 45, 1794-1801.

24. Gylling H \& Miettinen TA (1991) High cholesterol and fat intake have similar respective effects on serum cholesterol, LDL kinetics and cholesterol metabolism. Circulation 84, Suppl. II, 680. 